\title{
Lesión medular y embarazo: lo que el obstetra debe conocer
}

\author{
Andrés Sarmiento Rodríguez M.D.*; Alvaro Cano Quiñones M.D.**
}

\section{RESUMEN}

La relativa baja frecuencia de embarazos en mujeres con lesión medular hace que existan dudas sobre el manejo obstétrico de estas pacientes y se desconozcan los potenciales peligros y la forma de enfrentarlos. Cada día los programas de rehabilitación le brindan a estas pacientes un mejor nivel de vida y por ende nos enfrentaremos con mayor frecuencia a esta eventualidad por lo que es importante que el obstetra conozca el manejo de las principales complicaciones que en ocasiones, sin un manejo adecuado, pueden llegar a ser letales como lo es la Hiperreflexia autonómica. De esta manera se podrá ofrecer a la paciente una atención obstétrica adecuada y cuando el caso lo permita un parto por vía vaginal sin temor a exponerla a riesgos y complicaciones innecesarios.

PALABRAS CLAVES: Hiperreflexia autonómica, lesión medular, trabajo de parto, paraplejía.

\section{SUMMARY}

The reduced frequency of pregnancies in women with spinal cord lesions creates doubts about the obstetrical management of these patients and ignorance about the potential risks and how to prevent them. Every day de rehabilitation programs offer this patients better general health and we see more spinal cord-injured women in our practice; this makes it a priority for obstetricians to know how to provide an effective management of lethal complications as Autonomic Hiperreflexia. In this way we can offer the spinal cord-injured pregnant woman adequate care without unnecessary risks or complications.

KEY WORDS: Autonomic hiperreflexia, spinal cord injury, labor, Paraplegia.

\section{Introducción}

No es frecuente en nuestro medio la paciente que con secuelas de lesión medular consulte embarazada para control prenatal y/o atención del parto. Por lo general este tipo de paciente genera dudas terapéuticas para el obstetra. La tendencia habitual en el manejo de estas pacientes es la de un control prenatal rutinario, ofreciendo la opción quirúrgica a la paciente al momento del parto, sin considerar la vía vaginal como una alternativa viable.

En los últimos años en el mundo el número de pacientes en edad reproductiva con lesiones medulares ha aumentado en forma importante. Estas pacientes con secuelas de incapacidad física han recibido mayor atención y hoy en día se ha procurado ofrecerle un manejo integral a su problema. Esto no solamente incluye la atención misma del trauma si no que pretende involucrar a servicios de Rehabilitación, Terapia Física y Ocupacional, Psicología y eventualmente si la paciente se emba-

\footnotetext{
* Médico Ginecobstetra. Coordinador Académico de la Unidad de Medicina Materno Fetal Hospital Simón Bolivar. Universidad El Bosque. Director Científico, OBGYN Ltda.

** Médico Ginecobstetra. Unidad de Medicina Materno Fetal del Hospital Simón Bolivar. Ginecobstetra OBGYN Ltda.
}

raza, de Ginecobstetricia. Este enfoque busca ofrecer una mejor calidad de vida del paciente y pretende que las pacientes dejen de ser un lastre para su familia y la sociedad y se reintegren en una forma más adecuada a su vida cotidiana. Estos cambios han permitido que la sexualidad y el aspecto reproductivo en este grupo de pacientes tengan la importancia que se merecen. En los últimos años el embarazo se ha convertido en una causa frecuente de consulta en este grupo poblacional y es frecuente que el equipo multidisciplinario que los maneja no conozca a fondo las implicaciones de la gravidez en estas pacientes. El rehabilitador físico, el neurólogo, el anestesiólogo y el mismo obstetra frecuentemente desconocen detalles básicos de las opciones terapéuticas en estas pacientes.

En las siguientes líneas presentaremos el caso clínico de una paciente gestante con lesión medular postraumática. Posteriormente se practica la correspondiente revisión bibliográfica donde se evidencia como este grupo de pacientes precisa pautas individualizadas de manejo ante e intraparto.

\section{Caso clínico}

Paciente de 29 años $\mathrm{G}_{2} \mathrm{P}_{1}$ con paraplejía secundaria a trauma raquimedular a nivel de $T_{6}$ cinco meses atrás, quien ingresa al servicio de Ginecología y Obstetricia del 
Hospital Simón Bolívar con diagnóstico de embarazo de 38 semanas por última menstruación y actividad uterina irregular. Al examen de ingreso se encontró TA: 120/80, FC: 65 x', FUVLCDI con AU: $26 \mathrm{~cm}$, FCF: 150 x'; cuello corto con dilatación de $2 \mathrm{~cm}$ y borramiento del $60 \%$. Al examen neurológico se evidencia parálisis flácida de MsIs con ausencia de sensibilidad, hiperreflexia y vejiga neuropléjica. Se realiza ultrasonido obstétrico que reporta 35 semanas por biometría combinada con valoración biofísica satisfactoria. Se hace diagnóstico de Amenaza de Parto Pretérmino, Embarazo de 35 semanas y Paraplejía. Se envía a la Unidad de Medicina Materno Fetal (UMMF) donde se reevalúa el caso, y se practica nuevo ultrasonido obteniendo un diagnóstico de embarazo a término con RCIU. Es valorada por neurología confirmándose diagnóstico de Parálisis Flácida secundaria a Trauma Raquimedular y se recomienda cesárea como vía del parto. A las 24 horas de ingreso la paciente reinicia trabajo de parto, se realiza amniotomía temprana, se coloca analgesia peridural continua y refuerzo del trabajo de parto con oxitocina. Durante el trabajo de parto la paciente presenta cifras tensionales estables sin alteraciones de la frecuencia cardíaca ni síntomas autonómicos. Después de un trabajo de parto de 6 horas con una dinámica normal se pasa a sala de partos, se realiza una episiotomía medio lateral y se colocan espátulas de desprendimiento. Se obtiene recién nacido de $2.500 \mathrm{gm}$, con talla de $47 \mathrm{~cm}$, APGAR: 9/10, 9/10, 10/10, y Ballard de 38 semanas. Alumbramiento espontáneo a los 5 minutos. Puerperio de evolución normal y salida de la madre con el recién nacido a las 24 horas. Se continúa manejo por fisiatría.

\section{Discusión}

A pesar que en EUA anualmente 3.000 mujeres en edad reproductiva sufren lesiones medulares, son pocas las referencias bibliográficas disponibles en la literatura obstétrica acerca del manejo de estas pacientes. Las lesiones medulares aumentan el riesgo obstétrico de la madre pero el resultado perinatal generalmente es satisfactorio. Se ha observado en la población general que mujeres con alguna lesión medular se embarazan en un tiempo promedio de 6.5 años después de la lesión; siendo la edad promedio a su primer embarazo de 26.8 años. Estos datos no deben sorprender si tenemos en cuenta que la fertilidad en la mujer en edad reproductiva con lesión medular no está disminuida, al igual que la líbido, sus habilidades para sostener un coito, o dar a luz y criar un bebe.

La tasa de cesáreas en esta población alcanza al 43\%. Las indicaciones mas frecuentes son la iteratividad, presentaciones anormales y el trabajo de parto disfuncional.

Las complicaciones más frecuentes en pacientes embarazadas con lesión medular son:

\section{- Hiperreflexia Autonómica}

Se debe a la pérdida de la regulación central sobre el Sistema Nervioso Simpático (SNS) distal al nivel de la lesión. En respuesta a estímulos que ingresen a la médula distales al sitio de lesión se presenta hiperactividad simpática y parasimpática dada por descargas adrenérgicas y colinérgicas con control supraespinal disfuncional. Estímulos como la distensión vesical, las contracciones u.terinas, el dolor quirúrgico (como por ejemplo una cesárea), la manipulación del cérvix o la distensión del recto producen estimulación en masa de los aferentes simpáticos y parasimpáticos por debajo de la lesión desencadenando el cuadro. Se presenta hasta en un $75 \%$ de las pacientes con lesiones superiores a T-6.

Su diagnóstico se puede hacer tempranamente si comparamos los valores sistólico y diastólico de la presión arterial materna: elevaciones de más de 30 $\mathrm{mmHg}$ en la sistólica y $15 \mathrm{mmHg}$ en la diastólica, asociadas a cefalea pulsátil durante la contracción uterina o cualquier evento incitante (en ausencia de otra clínica o laboratorios sugestivos de preeclampsia), sugieren la presencia de hiperreflexia autonómica. Las manifestaciones clínicas más frecuentes incluyen hipertensión y cefalea pulsátil durante la contracción, bradicardia o taquicardia, arritmias cardíacas, diaforesis marcada, pilo erección y enrojecimiento de la piel proximal al nivel de la lesión.

El bloqueo peridural debe ser el tratamiento de elección. Este bloqueo es una alternativa profiláctica y terapéutica para estos casos. La morbilidad depende del grado de hipertensión, y se ha reportado hemorragia intracraneana durante el trabajo de parto atribuida a la hiperreflexia autonómica.

\section{- Infección de Vías Urinarias y Pielonefritis}

Se ha atribuido una mayor incidencia de infección urinaria en estas pacientes a la presencia de mayores residuos postmiccionales y a los procedimientos frecuentes de cateterización vesical a los que son sometidos. Sin embargo no se ha demostrado que este tipo de complicación sea más frecuente durante el embarazo que en la mujer con lesión medular no embarazada.

\section{- Complicaciones Tromboembólicas}

El decúbito prolongado y la falta de movilización de estas pacientes hacen que aumente el riesgo de eventos tromboembólicos. Nuevamente este riesgo es igual que en la población neurológicamente sana. No se recomienda la anticoagulación profiláctica a no ser que existan factores de riesgo o antecedentes presentes.

\section{- Trabajo de Parto Pretérmino}

Bastante discutido en diferentes publicaciones, se ha atribuido a la incapacidad de la madre para detectar el inicio de la áctividad uterina debido a su compromiso neurológico. Parece asociarse más a la mayor frecuencia de infecciones del tracto genitourinario y genitales, a la que están expuestas las mujeres con lesión medular. De todas maneras no se ha podido demostrar que la incidencia sea mayor que en la población control:

\section{- Ulceras de Presión, Sobreinfección y Sepsis}

Su incidencia es similar a la del resto de la población minusválida sin diferenciar por sexo. Depende esencialmente del cuidado de la paciente, de su educación y de la experiencia que tiene en su propia atención. Se puede 
favorecer debido al aumento de peso, la alteración del centro de gravedad y la dificultad para movilizarse que se presentan durante el embarazo.

\section{- Presentaciones Anormales, Obito Fetal y Mal- formaciones}

No se ha demostrado que la lesión neurológica favorezca las presentaciones anormales, o que los estudios radiológicos en estas pacientes (realizados con conocimiento previo del embarazo debido a su urgencia) aumenten la incidencia de obitos fetales o malformaciones respecto a la población sana.

- Otras complicaciones menos frecuentes son: anemia y disminución en el volumen corriente y en el volumen de reserva respiratoria

El pronóstico obstétrico y perinatal dependen del nivel de la lesión medular, observándose con mayor frecuencia las complicaciones severas como la hiperreflexia autonómica cuando la lesión se localiza en un nivel superior a T-6. Influyen también las condiciones que favorecen la infección localizada y la sepsis.

En general se prefiere el parto por vía vaginal. Si la inducción se realiza con oxitocina, el riesgo de hiperreflexia autonómica se incrementa por el aumento en el estímulo incitante. El manejo adecuado incluye anestesia peridural continua y acortamiento del expulsivo; generalmente no se requiere de episiotomía gracias a la relajación del piso pélvico dada por el bloqueo neurológico.
La analgesia a emplear depende de cada caso y su nivel de lesión medular: puede ser por bloqueos espinales o epidurales, anestesia general, narcóticos parenterales e incluso hasta un bloqueo pudendo. Nunca debe intentarse el parto vaginal o quirúrgico sin analgesia y/o anestesia.

El acortamiento del expulsivo es importante para reducir los estímulos que puedan desencadenar las complicaciones neurológicas. Se pueden emplear succionador por vacío, espátulas o fórceps según la experiencia del obstetra y la disponibilidad de la institución. En los diferentes estudios publicados no se ha encontrado diferencia significativa en la duración del trabajo de parto al comparar pacientes con lesión medular con aquellas sanas.

El resultado perinatal ha sido uniformemente bueno y satisfactorio, sin complicaciones obstétricas mayores cuando se toman las debidas precauciones y se detectan tempranamente las posibles complicaciones referidas anteriormente.

\section{Conclusiones}

Las pacientes con lesión medular pueden tener un embarazo y trabajo de parto normales, siempre y cuando el personal a cargo este familiarizado y conozca las posibles complicaciones de estas pacientes. En ausencia de contraindicaciones obvias, el manejo obstétrico siempre se debe preferir la vía vaginal para el parto. El uso de analgesia /anestesia es mandatorio para evitar complicaciones.

\section{BIBLIOGRAFIA}

1. Mc Gregor James A, Meeuwsen James. "Autonomic hiperreflexia: A mortal danger for spinal cord-damaged women in labor.", Am J Obstet Gynecol, February 1995, Vol 151 Number 3.

2. Baker E., Cardenas D., Benedetti $T$. "Risk associated with pregnancy in spinal cord-injured women", Obstet and Gynecol, Vol. 80. No. 3, Part 1, September 1992.

3. Berghella V., Spector T., Trauffer P., Jhonson D. "Pregnancy in patients with preexisting transverse myelitis", Obstet and Gynecol, Vol. 87, No. 5, Part 2, May 1996.

4. Baker. E., Cardenas D. "Pregnancy in spinal cord injured women", Arch Phys Med Rehabil, Vol 77, No 5: 501-507, May 1996.

5. Cross LL., Meythaler JM., Tuel SM., Cross Al. "Pregnancy, labor and delivery post spinal cord injury", Paraplejia, Vol 30 No 12: 890-902, December 1992.

6. Verduyn WH. "Pregnancy and delivery in tetraplegic women", J Spinal Cord Med, Vol 20, No 3: 371-374, July, 1997.
7. Crosby E., St Jean B., Reid B., Elliot RD. "Obstetrical anesthesia and analgesia in chronic spinal cord-injured women", Can J Anaesth, Vol 39, No 5, Part 1: 487-494, May 1992

8. Greenspoon JS.. Paul RH. "Paraplejia and quadriplegia: special considerations during pregnancy and labor and delivery". Am J Obstet Gynecol, Vol 155, No 4: 738-741, October 1986.

9. Colachois SC. "Autonomic hiperreflexia with spinal cord injury", J Am Paraplegia Soc, Vol 15 No 3: 171-186, July, 1992.

10. Wanner MB., Rageth CJ., Zach GA. "Pregnancy and autonomic hiperreflexia in patients with spinal cord lesions", Paraplejia, Vol 25, No 6: 482-490, Dec, 1987.

11. Gimovsky ML., Ojeda A., Ozaki R., Zerne S. "Management of autonomic hiperreflexia", Am J Obstet Gynecol, Vol 153, No 2: 223 224, Sept. 1985. 